\title{
An ordinal selection of stable sets in the sense of Hillas
}

Citation for published version (APA):

Vermeulen, A. J., \& Jansen, M. J. M. (2001). An ordinal selection of stable sets in the sense of Hillas. Journal of Mathematical Economics, 36(36), 161-167. https://doi.org/10.1016/S0304-4068(01)00073-8

Document status and date:

Published: 01/01/2001

DOI:

10.1016/S0304-4068(01)00073-8

Document Version:

Publisher's PDF, also known as Version of record

\section{Please check the document version of this publication:}

- A submitted manuscript is the version of the article upon submission and before peer-review. There can be important differences between the submitted version and the official published version of record.

People interested in the research are advised to contact the author for the final version of the publication, or visit the DOI to the publisher's website.

- The final author version and the galley proof are versions of the publication after peer review.

- The final published version features the final layout of the paper including the volume, issue and page numbers.

Link to publication

\footnotetext{
General rights rights.

- You may freely distribute the URL identifying the publication in the public portal. please follow below link for the End User Agreement:

www.umlib.nl/taverne-license

Take down policy

If you believe that this document breaches copyright please contact us at:

repository@maastrichtuniversity.nl

providing details and we will investigate your claim.
}

Copyright and moral rights for the publications made accessible in the public portal are retained by the authors and/or other copyright owners and it is a condition of accessing publications that users recognise and abide by the legal requirements associated with these

- Users may download and print one copy of any publication from the public portal for the purpose of private study or research.

- You may not further distribute the material or use it for any profit-making activity or commercial gain

If the publication is distributed under the terms of Article $25 \mathrm{fa}$ of the Dutch Copyright Act, indicated by the "Taverne" license above, 


\title{
An ordinal selection of stable sets in the sense of Hillas
}

\author{
A.J. Vermeulen*, M.J.M. Jansen \\ Department of Quantitative Economics, Faculty of Economics and Business Administration, \\ Maastricht University, P.O. Box 616, 6200 Maastricht, MD, The Netherlands
}

Received 24 March 2000; accepted 19 July 2001

\begin{abstract}
In this paper, it is shown in an example that the original definition of stable sets in Hillas [Econometrica 58 (1990) 1365-1391] does not satisfy (an even slightly weakenened version of) the invariance condition proposed in Mertens [Ordinality in noncooperative games, Core Discussion Paper 8728, CORE Louvain de la Neuve, Belgium, 1987]. However, it is also shown that the basic stability condition of Hillas underlying his definition of stable sets does admit a selection that is invariant in the strong sense, and even ordinal. (C) 2001 Elsevier Science B.V. All rights reserved.
\end{abstract}

Keywords: Game theory; Stable sets; Invariance

\section{Introduction}

The development of the theory of stable sets was initiated by the paper of (Kohlberg and Mertens, 1986). Their aim was to construct a solution ${ }^{1}$ that satisfies a number of conditions they considered to be absolutely essential for any reasonable solution.

One of these conditions is invariance. Originally, at least in the paper of Kohlberg and Mertens, a solution was said to be (KM-)invariant if it only depends on the reduced normal form of the game. They even argued that it should only depend on the reduced strategic form of the game. To be a bit more formal, the identification of payoff-equivalent strategy profiles of a game induces a natural projection from the space of strategy profiles of the game onto the space of strategy profiles of its reduced strategic form. A solution is now said

\footnotetext{
* Corresponding author. Fax: +31-4338-84874.

E-mail address: D.Vermeulen@ke.unimaas.nl (A.J. Vermeulen).

${ }^{1}$ In this context, a solution is a rule that assigns to each normal form game a collection of sets of strategy profiles of that game, the solution sets.
} 
to be KM-invariant if for any two games having the same reduced strategic form the images of the solution sets of each of these games coincide under their associated projections onto their mutual reduced strategic form.

In an attempt to construct a solution that satisfies all conditions required in the paper of (Kohlberg and Mertens, 1986) (Hillas, 1990) defined a new type of stable sets. Basically he required sets of equilibria to be robust against any sufficiently small perturbation of the best reply correspondence. We will refer to sets that are robust in this sense as BR-sets. Now the usual way to extract a reasonable solution from such a basic definition was to consider those sets that are minimal within the collection of all sets of equilibria that are robust in the sense under consideration. However, it is not clear whether the resulting solution is KM-invariant or not.

Therefore, Hillas also applied a technique that was already used by Kohlberg and Mertens in their definition of hyperstable sets. Although the formulation of this technique is somewhat ambiguous in both papers, the idea can best be described as follows. Given a (normal form) game, consider the equivalence class of all (normal form) games that have the same reduced strategic form as the given game. Then, within the strategy space of the reduced strategic form game, there is at least one set that, for any game in the equivalence class, is the projection of some BR-set of that game (namely, the whole strategy space itself). Consider the collection of sets within this strategy space that also have this property and select the minimal ones w.r.t. set inclusion in this collection. Now, for the given (normal form) game the solution sets are defined to be those BR-sets that project onto one of these minimal sets in the strategy space of the reduced strategic form game. It is fairly straightforward to show that the resulting solution is KM-invariant.

In (Mertens, 1987), the author gave a further strengthening of the invariance condition. He argued that a reasonable solution should be ordinal and showed that a solution is ordinal if it is both invariant in a strong sense and admissible-best-reply invariant (abr-invariant). See also (Vermeulen and Jansen, 2000).

In this paper, we will first give an example that shows that the solution proposed by Hillas is not invariant in the strong sense. Although we use a slightly different way to construct a KM-invariant solution given the basic definition, the same example also works for Hillas' original definition. We deviate from the original definition simply because it would take too much time to work out the details of the original definition and because the resulting differences between Hillas' definition and the one we use are not relevant for the arguments given in the example. As already observed by (Mertens, 1987), the real culprit that causes lack of invariance is the minimality condition.

Secondly, we will show that there is a quite natural way to select BR-sets such that the resulting solution is both invariant in the strong sense and abr-invariant, and therefore also ordinal.

\section{Preliminaries}

A normal form game with player set $N$ is a pair $\Gamma=\langle A, u\rangle$ such that $A:=\prod_{i \in N} A_{i}$ is the product of non-empty and finite pure strategy sets $A_{i}$ and $u=\left(u_{i}\right)_{i \in N}$ is an $N$-tuple of payoff functions $u_{i}: A \rightarrow \mathbb{R}$. 
For a strategy profile $x=\left(x_{i}\right)_{i \in N} \in \Delta:=\prod_{j \in N} \Delta_{j}$, the expected payoff of player $i$ is denoted by $u_{i}(x)$. Here, $\Delta_{i}$ is the set of probability distributions on $A_{i}$.

A solution is a map $\sigma$ that assigns to each game $\Gamma$ a collection $\sigma(\Gamma)$ of (non-empty and) closed subsets (called solution sets) of the strategy space of $\Gamma$. In (Mertens, 1987), it is shown that a solution is ordinal if it is both invariant and admissible-best-reply invariant (abr-invariant). Since these last two conditions are in fact the ones that will be used in this paper, we will first recall their definitions.

A solution is called abr-invariant if the solution sets coincide for those (so-called abrequivalent) games for which the best replies against a completely mixed strategy profile coincide.

In order to define invariance, let $\Gamma$ and $\Gamma^{\prime}$ be two games. A map $f=\left(f_{i}\right)_{i \in N}$ from $\Delta^{\prime}$ to $\Delta$ is called a reduction map from $\Gamma^{\prime}$ to $\Gamma$ if each map $f_{i}$ is linear and onto from $\Delta_{i}^{\prime}$ to $\Delta_{i}$ and the payoff function $u_{i}^{\prime}$ equals the composition $u_{i} \circ f$ of the payoff function $u_{i}$ with $f$. Now a solution $\sigma$ is called invariant if for any reduction map $f$ from a game $\Gamma^{\prime}$ to a game $\Gamma$, the set $f(T)$ is a solution set of the game $\Gamma$ for every solution set $T$ of the game $\Gamma^{\prime}$ and, conversely, for every solution set $S$ of the game $\Gamma$ the set $f^{-1}(S)$ is equal to the union over all solution sets $T$ of the game $\Gamma^{\prime}$ for which $f(T)=S$.

Following (Hillas, 1990), we define best-reply sets (BR-sets) as follows. A closed set of strategy profiles is called a BR-set if any neighborhood of that set contains at least one fixed point of any compact and convex-valued upper hemicontinuous correspondence whose pointwise Hausdorff distance to the best reply correspondence is sufficiently small. A BR-set $S$ is called extensible (cf. (Vermeulen et al., 1995)) if $f^{-1}(S)$ is also a BR-set for any reduction map $f$.

\section{An example}

In this section, we will show that the (slightly changed ${ }^{2}$ ) definition of stability in the sense of (Hillas, 1990) does not satisfy invariance. In fact we will consider two ways to define solution sets using BR-sets. On the one side we consider the minimal extensible BR-sets as solution sets and on the other hand the minimal BR-sets.

We will show now that the first solution - although it does satisfy the (weaker) type of invariance mentioned in the paper of (Kohlberg and Mertens, 1986) — is not invariant in the sense of the previous section. The same example can also be used to show that neither the second solution is invariant.

Consider the $3 \times 4$-bimatrix game

$$
\Gamma^{\prime}:\left[\begin{array}{llll}
(0,2) & (4,0) & (2,1) & (2,1) \\
(4,0) & (0,2) & (2,1) & (2,1) \\
(3,2) & (3,2) & (3,2) & (3,2)
\end{array}\right]
$$

\footnotetext{
${ }^{2}$ This change is a result of both a change in the definition of invariance and an ambiguity in the original definition of stable sets. A justification of these adjustments is given in the introduction.
} 
and let $T$ be the Cartesian product of the one-point set $\{(0,0,1)\}$ (for player 1$)$ and the set in the strategy space of player 2 consisting of the following three line segments: the first one from $(3 / 4,1 / 4,0,0)$ to $(1 / 16,5 / 16,1 / 2,1 / 8)$, the second one from $(1 / 16,5 / 16,1 / 2,1 / 8)$ to $(3 / 8,1 / 8,1 / 2,0)$ and the third one from $(3 / 8,1 / 8,1 / 2,0)$ to $(1 / 4,3 / 4,0,0)$. The first two line segments are depicted below by the coarsely dotted lines and the third one is the line segment in the front face of the tetrahedron.

The following is the strategy space of player 2 :

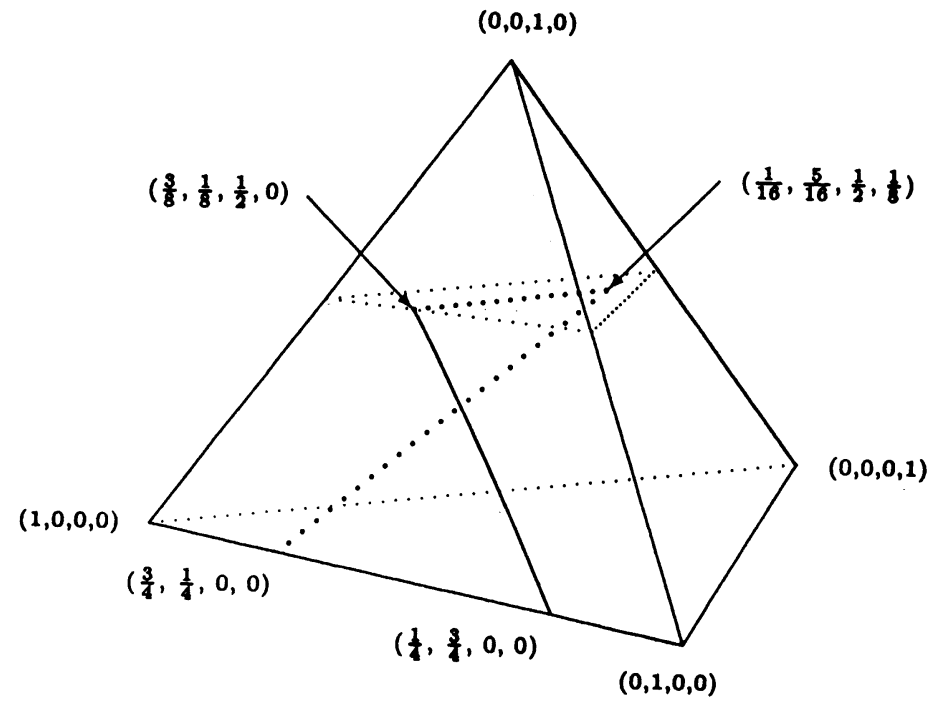

It can be shown that $T$ is a stable set in the sense of (Mertens, 1989) (and therefore a BR-set). By the proof of Proposition 11 of (Mertens, 1987), it follows that, for any reduction map $f$ from a game $\Gamma^{\prime \prime}$ to $\Gamma^{\prime}, f^{-1}(T)$ is a BR-set. So $T$ is an extensible BR-set. We will show that $T$ is also minimal. To this end, note that the two-point set

$$
S:=\{(0,0,1)\} \times\left\{\left(\frac{3}{4}, \frac{1}{4}, 0,0\right),\left(\frac{1}{4}, \frac{3}{4}, 0,0\right)\right\}
$$

is the only stable set in the sense of Kohlberg and Mertens of the game $\Gamma^{\prime}$ that is contained in $T$. However, by Proposition 3 of (Hillas, 1990), we know that any stable set in the sense of Hillas is connected and $T$ is the only connected subset of $T$ that contains $S$. Hence, $T$ must be minimal.

Now consider the $3 \times 3$-bimatrix game

$$
\Gamma:\left[\begin{array}{lll}
(0,2) & (4,0) & (2,1) \\
(4,0) & (0,2) & (2,1) \\
(3,2) & (3,2) & (3,2)
\end{array}\right]
$$

and the reduction map $f$ from $\Gamma^{\prime}$ to $\Gamma$ with $f_{1}$ equal to identity and $f_{2}$ is the linear map

$$
f_{2}\left(q_{1}, q_{2}, q_{3}, q_{4}\right)=\left(q_{1}+\frac{1}{2} q_{4}, q_{2}+\frac{1}{2} q_{4}, q_{3}\right)
$$


It is easy to check that the set $M:=M_{1} \cup M_{2}$ with

$$
\begin{aligned}
& M_{1}:=\{(0,0,1)\} \times\left\{(1-s)\left(\frac{3}{4}, \frac{1}{4}, 0\right)+s\left(\frac{1}{3}, \frac{1}{3}, \frac{1}{3}\right) \mid 0 \leq s \leq 1\right\} \\
& M_{2}:=\{(0,0,1)\} \times\left\{(1-s)\left(\frac{1}{3}, \frac{1}{3}, \frac{1}{3}\right)+s\left(\frac{1}{4}, \frac{3}{4}, 0\right) \mid 0 \leq s \leq 1\right\}
\end{aligned}
$$

is a proper subset of $f(T)$. Moreover, $M$ is a minimal extensible BR-set by the same line of reasoning used above. Hence, $f(T)$ is not a minimal extensible BR-set which shows that the solution that assigns to each game its minimal extensible BR-sets is not invariant.

\section{The ordinal selection}

Since the solution that assigns to a game its collection of extensible BR-sets is, as we will also see in this section, invariant and abr-invariant the above example suggests that the lack of invariance of stable sets in the minimal extensible BR-sets is mainly due to the minimality condition. Such an incompatibility of minimality with invariance was already noted by (Mertens, 1989). Leaving out the entire minimality condition is not very satisfactory though, since the solution we, thus, get violates several other requirements of the Kohlberg-Mertens program.

In this section, we will apply a relaxation of the minimality condition similar to the one used by Mertens. In fact we will consider the solution $\tau$ that assigns to a game the collection of extensible BR-sets that are a connected subset of the set of perfect equilibria of the game in question. From now on we will simply refer to the elements of $\tau(\Gamma)$ as solution sets of $\Gamma$. We will show that this way of selecting BR-sets yields an invariant and abr-invariant solution that also satisfies the other relevant properties of Hillas' solution. Then, by Theorem 2 of (Mertens, 1987), it follows that $\tau$ is ordinal.

Using the fact that minimal BR-sets are connected sets of perfect equilibria it can be showed (as in Theorems 6 and 7 of (Vermeulen et al., 1995)) that minimal extensible BR-sets are also connected sets of perfect equilibria. Hence, every game has at least one solution set and the solution $\tau$ is well-defined.

Next, we will show the following theorem.

Theorem 1. The solution $\tau$ is invariant.

Proof. Since a reduction map $f$ from a game $\Gamma^{\prime}$ to a game $\Gamma$ preserves closedness, connectedness, perfectness, BR-sets and extendibility (cf. Proposition 1 of (Vermeulen et al., $1995)), f(T)$ is a solution set if $T$ is. Since $f^{-1}$ has the same preservation properties $f^{-1}(S)$ is a solution set if $S$ is.

Theorem 2. The solution $\tau$ is abr-invariant.

Proof. Let $\Gamma$ and $\Gamma^{*}$ be two abr-equivalent games and let $S$ be a solution set of the game $\Gamma^{*}$. Then, by Theorem 3 of (Vermeulen and Jansen, 1997), it must be a closed and connected set of perfect equilibria of the game $\Gamma$. In order to show that $S$ is also an extensible set of the game $\Gamma$, take a reduction map $f$ from a game $\Gamma^{\prime}$ to $\Gamma$. Now let $\left(\Gamma^{*}\right)^{\prime}$ be the game 
with the same strategy space as $\Gamma^{\prime}$ for which the payoff function of a player equals the composition of the payoff function of that player in the game $\Gamma^{\prime}$ and $f$. Since each $f_{i}$ preserves best replies (by Lemma 1 of (Vermeulen and Jansen, 1997)), the games $\left(\Gamma^{*}\right)^{\prime}$ and $\Gamma^{\prime}$ are abr-equivalent. Since, moreover, $f$ is a reduction map from $\left(\Gamma^{*}\right)^{\prime}$ to $\Gamma^{*}, f^{-1}(S)$ is a BR-set of the game $\left(\Gamma^{*}\right)^{\prime}$. This implies that $f^{-1}(S)$ is also a BR-set of the game $\Gamma^{\prime}$. Hence, $S$ is an extensible set for $\Gamma$ and, therefore, a solution set of the game $\Gamma$. By symmetry the proof is complete.

Finally, we will show that the solution $\tau$ satisfies all the relevant properties of Hillas' solution. First of all solutions sets are connected sets of perfect equilibria by definition and a solution set — being a BR-set — contains a proper equilibrium.

In order to show that the solution $\tau$ is independent of inadmissible strategies, we need some terminology. The deletion of a pure strategy, say $d$, of the set of pure strategies of some player $i$ in a game $\Gamma$ induces a game $\Gamma^{*}$ for which the payoff function of each player is the obvious restriction of the original payoff function. Furthermore, a subset $S$ of $\Delta$ induces the subset $S^{*}$ of the strategy space $\Delta^{*}$ of $\Gamma^{*}$ obtained by skipping the $d$ th coordinate of $x_{i}$ in every strategy profile $x \in S$ for which this coordinate equals zero.

A solution is independent of inadmissible strategies if for any solution set $S$ of a game $\Gamma$ for which there is a pure strategy $d$ that is not an admissible best reply against $S$ the set $S^{*}$ (corresponding with the deletion of $d$ ) contains a solution set of the game $\Gamma^{*}$. Here, a pure strategy $b$ of a player is called an admissible pure best reply against $S$ if there exists a strategy profile $x$ in $S$ against which $b$ is an admissible best reply. Note that for a BR-set $S$, $S^{*}$ is a non-empty BR-set. Now we have the following theorem.

\section{Theorem 3. The solution $\tau$ is independent of inadmissible strategies.}

Proof. Let $S$ be a solution set of the game $\Gamma$, and suppose that the pure strategy $d$ of player $j$ is not an admissible best reply against $S$.

1. We will show that $S^{*}$ is an extensible BR-set of the game $\Gamma^{*}$.

So, let $f$ be a reduction map from a game $\left(\Gamma^{*}\right)^{\prime}$ to $\Gamma^{*}$. Now let $\Gamma^{\prime}$ be the game obtained from $\left(\Gamma^{*}\right)^{\prime}$ by adding the strategy $d$ as a pure strategy for player $j$ and where the payoff function of a player equals the composition of the payoff function of that player in the game $\left(\Gamma^{*}\right)^{\prime}$ and the reduction map $g$ from $\Gamma^{\prime}$ to $\Gamma$ that coincides with $f$ and with $g_{j}(d)=d$. Then $g^{-1}(S)$ is a BR-set of the game $\Gamma^{\prime}$. So, by Lemma 6 of (Vermeulen et al., 1995), $d$ is not an admissible best reply against $g^{-1}(S)$. Since, by Proposition 4 of (Hillas, 1990), $\left[g^{-1}(S)\right]^{*}$ contains a BR-set of the game $\left(\Gamma^{*}\right)^{\prime},\left[g^{-1}(S)\right]^{*}=f^{-1}\left(S^{*}\right)$ is a BR-set of the game $\left(\Gamma^{*}\right)^{\prime}$. Hence, $S^{*}$ is an extensible BR-set of $\Gamma^{*}$.

2. Since - as we observed at the beginning of this section - a BR-set contains a minimal BR-set and such a set is a solution set, the proof is complete.

\section{References}

Hillas, J., 1990. On the definition of the strategic stability of equilibria. Econometrica 58, 1365-1391.

Mertens, J.F., 1987. Ordinality in noncooperative games, Core Discussion Paper 8728, CORE Louvain de la Neuve, Belgium. 
Kohlberg, E., Mertens, J.F., 1986. On strategic stability of equilibria. Econometrica 54, 1003-1037.

Vermeulen, A.J., Jansen, M.J.M., Potters, J.A.M., 1995. Making solutions invariant, Report 9519, Department of Mathematics, University of Nijmegen, Nijmegen.

Mertens, J.F. 1989. Stable equilibria — a reformulation. Part I. Definitions and basic properties. Mathematics of Operations Research 14, 575-625.

Vermeulen, A.J., Jansen, M.J.M., 1997. On the invariance of solutions of finite games. Mathematical Social Sciences 33, 251-267.

Vermeulen, A.J., Jansen, M.J.M., 2000. Ordinality of solutions of noncooperative games. Journal of Mathematical Economics 33, 13-34. 